\title{
Nipple Adenoma Treated with Nipple Preservation in Young Female Patient: A Report of 2 Cases
}

\author{
Soo Young Lim, M.D. ${ }^{*}$, Jung Han Yoon, M.D., Ph.D. ${ }^{1 *}$, Yong Min Na, M.D. ${ }^{1}$, Young Jae Ryu, M.D. ${ }^{1}$, \\ Hyo Soon Lim, M.D., Ph.D. ${ }^{2}$, Ji Shin Lee, M.D., Ph.D. ${ }^{3}$, Jin Seong Cho, M.D. ${ }^{4}$, Min Ho Park, M.D., Ph.D. ${ }^{1}$ \\ Departments of ${ }^{1}$ Surgery, ${ }^{2}$ Radiology, ${ }^{3}$ Pathology, Chonnam National University Medical School and Chonnam National University Hwasun Hospital, \\ Jeollanam-do; ${ }^{4}$ Department of Surgery, Chonnam National University Medical School and Chonnam National University Hospital, Gwangju, Korea
}

Adenoma of the nipple is a rare benign type of breast that occurs most often in middle-aged women with nipple discharge, skin erosion in addition to crusting, inflammation, and pain. It can be clinically confused with Paget's disease or breast cancer precursor lesions, such as ductal carcinoma in situ low grade. The treatment of choice for nipple adenoma is complete excision of the tumor. However in younger women, nipple preservation is required. We present two cases of nipple adenoma that were completely removed with nipple preservation.

Key Words: Adenoma, Nipples, Preservation

\section{INTRODUCTION}

Nipple adenoma is a rare breast tumor that represents benign proliferation of lactiferous ducts [1-3]. It occurs most often in the 4 th and 5 th decades of life $[2,4]$. The clinical presentation includes nipple discharge, skin erosion, crusting, inflammation, erythema, itching, and/ or associated pain of the nipple region [5]. It can be confused with other lesions such as Paget's disease, low-grade ductal carcinoma in situ (DCIS), tubular carcinoma, and infiltrating syringomatous adenoma [6]. Complete excision of the tumor is the standard treatment of the nipple adenoma. According to tumor size and location, nipple-preserving tumor resection or total resection of the nipple-areolar complex or sometimes mastectomy can be performed [7-9]. Here we report two cases of nipple adenoma that were successfully treated after complete excision with nipple preservation.

Our Institutional Review Board approved this study (CNUHH2021-146) and waived the requirement for obtaining informed consent.

Correspondence: Min Ho Park, M.D., Ph.D.

Department of Surgery, Chonnam National University Medical School and Chonnam National University Hwasun Hospital, 322 Seoyang-ro, Hwasun-eup, Hwasun-gun, Jeollanam-do 58128, Korea

Tel: +82-61-379-7646, Fax: +82-61-379-7661, E-mail: mhpark@chonnam.ac.kr

*These authors contributed equally to this work.

Received: Oct 12, 2021 Revised: Oct 23, 2021 Accepted: Oct 31, 2021

\section{CASE REPORT}

\section{Case 1}

A 33-year-old female came to the hospital with a sessile mass at the top of her left nipple. The patient complained of bloody nipple discharge with a palpable mass on the left nipple for 3 weeks before her first visit to the clinic. It has been proven to be a nipple adenoma on excisional biopsy. Ultrasonography showed no other specific lesion, except the mass on the left nipple (Figure 1A). The patient underwent a nipple-mass wide excision under general anesthesia. There was about a 1.2-cm-sized cauliflower-shaped whitish-colored mass on the tip of the nipple (Figure 2A). Incision was performed on normal tissue around the mass at the nipple, and the subareolar ducts were resected together. Complete excision of the mass with a clear margin was performed without total excision of the nipple (Figure 2B, C). Pathologic findings showed an adenomatous pattern of the nipple adenoma. The duct epithelial cells proliferated and formed small tubules, which mixed extensively in the fibrous stroma (Figure 3A). The patient had no recurrence until 3 years after surgery (Figures 1B, 2D).

\section{Case 2}

A 26-year-old female had complained of skin changes and bloody discharge at the right nipple. A polypoid mass covered $90 \%$ of the tip of the right nipple. It was proven to be a nipple adenoma on punch bi- 

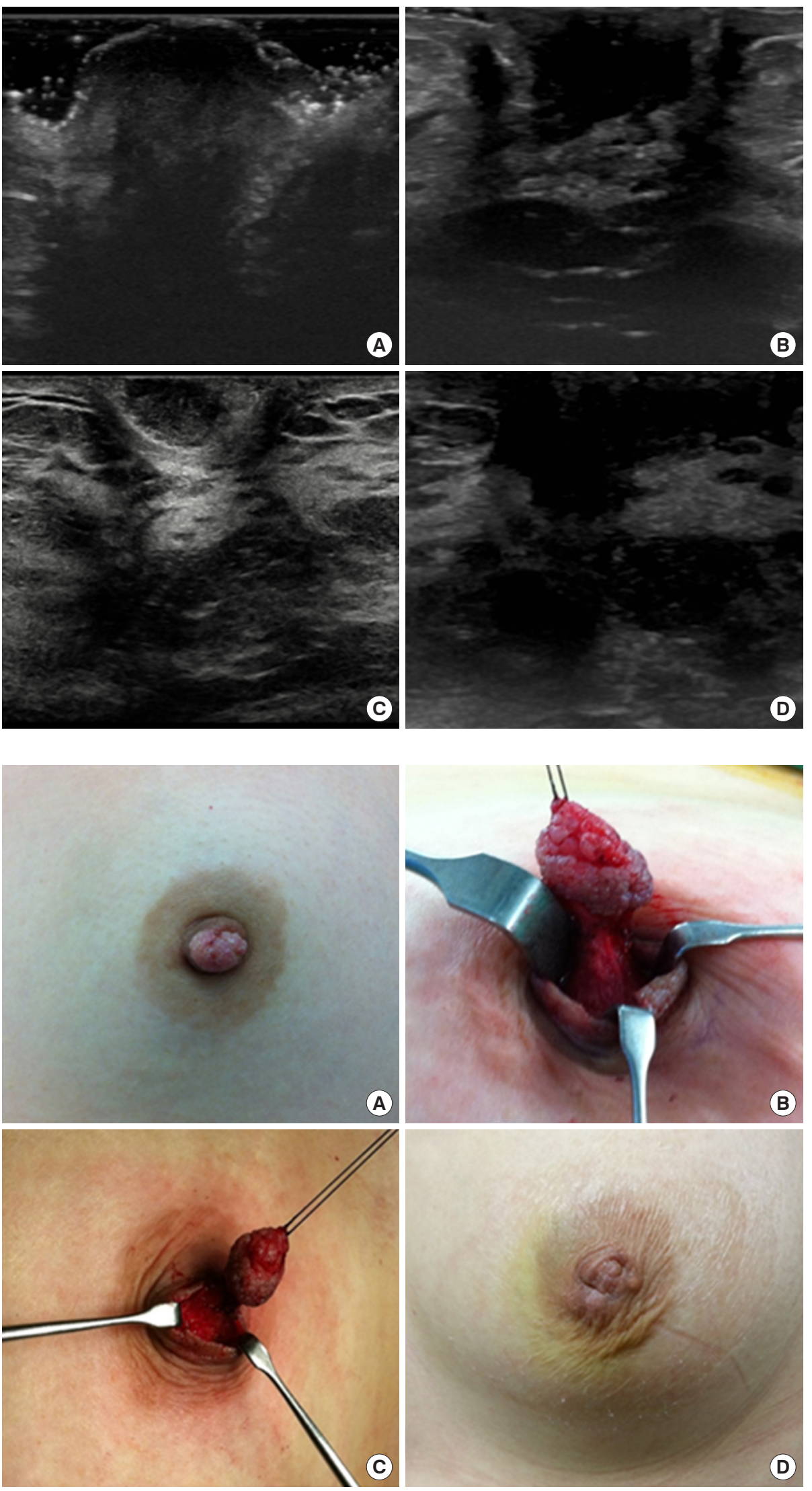

Figure 1. Ultrasonographic findings. (A) Preoperative ultrasonography (case 1). Irregular-shaped hypoechoic lesion on the left nipple. (B) Postoperative ultrasonography (case 1). Surgery after 3-year follow-up ultrasonography shows post-excisional change and no sign of local recurrence observed. (C) Preoperative ultrasonography (case 2). Enhancing lesion at the right subareolar area directly under the nipple with diffuse duct ectasia. (D) Postoperative ultrasonography (case 2). Six months after excision, follow-up ultrasonography does not show any sign of recurrence.

Figure 2. Preoperative, intraoperative, and postoperative images of case 1. (A) Nipple image. Cauliflower shaped whitish color mass on the top of the nipple. The mass was fragile and easily bled. (B, C) Partial excision of the nipple including tumor with nipple preservation. (D) Postoperative image. 

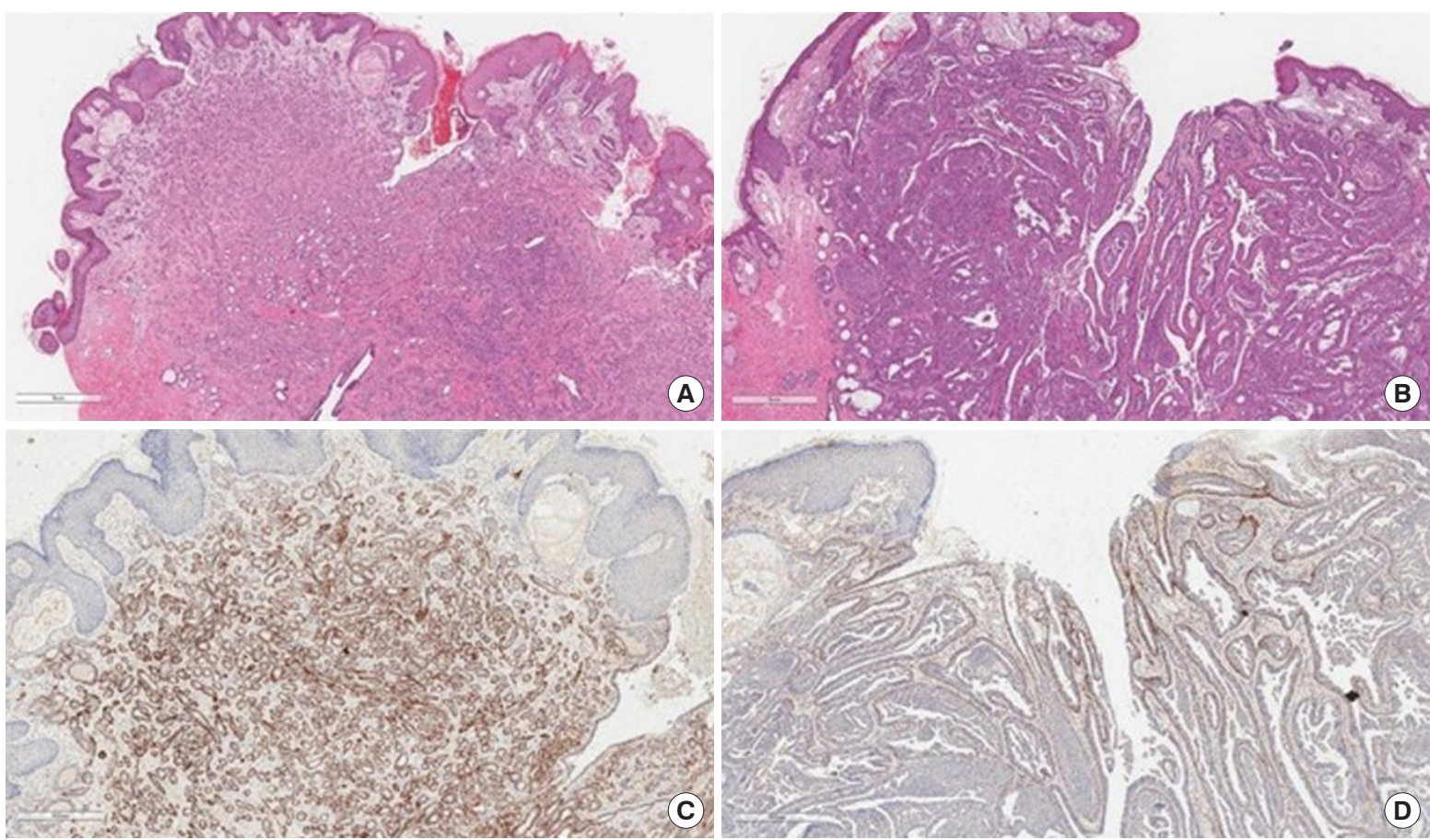

Figure 3. Histopathological findings of nipple adenoma. (A) Adenomatous pattern (case 1), (B) papillomatous pattern with usual ductal hyperplasia (case 2), $(C, D)$ smooth muscle myosin heavy chain immunostaining (C: case $1, D$ : case 2$)(A, B: H \& E, \times 2 ; C, D$ : immunostain, $\times 4)$.

opsy. Ultrasonography showed an enhancing lesion in the right subareolar area directly under the nipple with diffuse duct ectasia (Figure 1C). There was a 5-mm-sized hypoechoic nodule on the upper-inner side of the subareolar area. There were no other specific lesions in either breast. The patient underwent wide excision of the right nipple under general anesthesia. The base of the mass was broad and spanned over $1 \mathrm{~cm}$ in the right nipple. Surgery was performed with an elliptical incision, and the subareolar ducts were also partially resected, and the nipple was cosmetically preserved well. On histological examination, the tumor appeared as a nipple adenoma with a papillomatous pattern and usual ductal hyperplasia (Figure 3B). Follow-up ultrasonography after 6 months showed no detectable mass (Figure 1D).

\section{DISCUSSION}

Nipple adenoma is a florid, benign proliferation of the nipple ductal epithelium. Erosive adenomatosis of the nipple, florid papillomatosis of the nipple ducts, nipple duct adenoma and papillary adenoma of the nipple are alternative names of nipple adenoma [1-3]. In the World Health Oragnization classification of breast tumors, nipple adenoma is defined as "a compact proliferation of small tubules lined by epithelial and myoepithelial cells, with or without proliferation of the epithelial component, around the collecting ducts of the nipple [6,7]."

It usually occurs in middle-aged women, but can occur at any age, and at less than 5\% it can arise in men [2,4]. Patients with nipple adenoma with a palpable nodule at the nipple and skin erosion, with or without nipple discharge. The symptoms appear unilaterally in most cases, but bilateral diseases have also been reported. Clinically, it may resemble Paget's disease and squamous cell carcinoma of the nipple, and pathologically, it may be confusing because of the differential diagnosis of low-grade DCIS, tubular carcinoma, tubular adenoma, ductal adenoma, solitary central papilloma, and infiltrating syringomatous adenoma [6].

The gold standard for the diagnosis of nipple adenoma is pathological examination after complete excision [6]. However, tissue biopsy prior to surgical excision is recommended, fine-needle aspiration, $\mathrm{cu}-$ rettage cytology, touch prep cytology and dermatoscopic examination can be used for diagnosis. Imaging studies, such as ultrasonography and mammography, usually have limitations in diagnosing nipple adenoma, because of the similarity of tissue density between the nipple and the surrounding skin. However some studies have reported that 
homogenous echogenicity and hypervascularity on ultrasonography can help recognize nipple adenomas [5].

The pathological features of nipple adenomas have very diverse patterns. Most nipple adenomas show ductal proliferation of glandlike structures within the nipple stroma. Our cases also showed ductal epithelial cell proliferation and small tubular formation in the fibrous stroma. Immunohistochemical staining is also useful for revealing the presence of myoepithelial cells [5]. In our cases, myoepithelial cells of the outer layer were positive for immunohistochemical staining for smooth muscle myosin heavy chain, supporting the benignancy of the nipple adenoma (Figure 3C, D).

The treatment of choice for nipple adenoma is complete surgical excision of the tumor. Incomplete resection can lead to a local recurrence. However, many young women usually do not want total resection of the nipple, and this is not an essential treatment. According to the location and size of the tumor, partial nipple resection including the tumor can be considered for nipple preservation. Because the tumor is located in the nipple, the decision of the surgical plan can be considerably difficult but important [9].

Wang et al. [9] reported 12 cases of nipple adenoma treated with surgical resection. Among the 12 cases, partial nipple resections were performed in 11 cases, and whole nipple removal was performed in 1 case. In all patients, there were no recurrences despite nipple preservation. We performed nipple-preserving surgery in both cases with small tumor located on the tip of the nipple. Consequently, nipple preservation can be considered in young female patients with small tumor size and, location on the tip of nipple, while a patient with a large tumor or invading the base of nipple or old age should be considered for en bloc resection of the nipple, if necessary, with breast tissue resection.

Nipple adenoma is a rare benign tumor that can be easily treated with complete surgical excision. It is often mistaken for Paget's disease or misinterpreted as breast carcinoma pathologically, so clinicians should be aware of this tumor and be able to make a proper diagnosis and treatment.

\section{CONFLICT OF INTEREST}

The authors declare that they have no competing interests.

\section{REFERENCES}

1. Goldman RL, Cooperman H. Adenoma of the nipple: a benign lesion simulating carcinoma clinically and pathologically. Am J Surg 1970;119:322-5.

2. Handley RS, Thackray AC. Adenoma of nipple. Br J Cancer 1962; 16:187-94.

3. Jones DB. Florid papillomatosis of the nipple ducts. Cancer 1955;8: 315-9.

4. Tsushimi T, Enoki T, Takemoto Y, Harada E, Hayashi M, Furuya T, et al. Adenoma of the nipple, focusing on the contrast-enhanced magnetic resonance imaging findings: report of a case. Surg Today 2011;41:1138-41.

5. Spohn GP, Trotter SC, Tozbikian G, Povoski SP. Nipple adenoma in a female patient presenting with persistent erythema of the right nipple skin: case report, review of the literature, clinical implications, and relevancy to health care providers who evaluate and treat patients with dermatologic conditions of the breast skin. BMC Dermatology 2016;16:1-10.

6. Di Bonito M, Cantile M, Collina F, D’Aiuto M, Liguori G, De Cecio $\mathrm{R}$, et al. Adenoma of the nipple: a clinicopathological report of 13 cases. Oncol Lett 2013;7:1839-42.

7. Fujii T, Yajima R, Morita H, Yamaguchi S, Tsutsumi S, Asao T, et al. Adenoma of the nipple projecting out of the nipple: curative resection without excision of the nipple. World J Surg Oncol 2014;12:1-4.

8. Kijima Y, Matsukita S, Yoshinaka H, Owaki T, Aikou T. Adenoma of the nipple: report of a case. Breast Cancer 2006;13:95-9.

9. Wang C, Wang X, Ma R. Diagnosis and surgical treatment of nipple adenoma. ANZ J Surg 2014;85:444-7. 